\title{
Reconnection current sheet structure in a turbulent medium
}

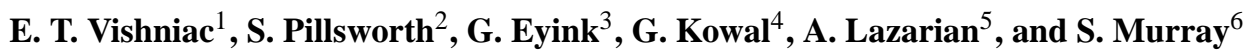 \\ ${ }^{1}$ Department of Physics and Engineering Physics, University of Saskatchewan, Saskatoon SK S7N 5E2, Canada \\ ${ }^{2}$ Department of Physics and Astronomy, McMaster University, Hamilton ON L8S 4M1, Canada \\ ${ }^{3}$ Department of Applied Mathematics and Statistics, Johns Hopkins University, 3400 N. Charles St., Baltimore, \\ MD 21218, USA \\ ${ }^{4}$ Instituto de Astronomia, Geofísica e Ciências Atmosféricas, Universidade de São Paulo, Rua do Matão, \\ 1226 - Cidade Universitária, CEP 05508-090, São Paulo/SP, Brazil \\ ${ }^{5}$ Department of Astronomy, University of Wisconsin, 475 North Charter Street, Madison, WI 53706, USA \\ ${ }^{6}$ Department of Mechanical Engineering, McMaster University, Hamilton ON L8S 4M7, Canada
}

Correspondence to: E. T. Vishniac (ethan.vishniac@usask.ca)

Received: 6 December 2011 - Revised: 17 August 2012 - Accepted: 22 September 2012 - Published: 8 November 2012

\begin{abstract}
In the presence of turbulence, magnetic field lines lose their dynamical identity and particles entrained on field lines diffuse through space at a rate determined by the amplitude of the turbulence. In previous work (Lazarian and Vishniac, 1999; Kowal et al., 2009; Eyink et al., 2011) we showed that this leads to reconnection speeds which are independent of resistivity. In particular, in Kowal et al. (2009) we showed that numerical simulations were consistent with the predictions of this model. Here we examine the structure of the current sheet in simulations of turbulent reconnection. Laminar flows consistent with the SweetParker reconnection model produce very thin and well ordered currents sheets. On the other hand, the simulations of Kowal et al. (2009) show a strongly disordered state even for relatively low levels of turbulence. Comparing data cubes with and without reconnection, we find that large scale field reversals are the cumulative effect of many individual eddies, each of which has magnetic properties which are not very different from turbulent eddies in a homogeneous background. This implies that the properties of stationary and homogeneous MHD turbulence are a reasonable guide to understanding turbulence during large scale magnetic reconnection events. In addition, dissipation and high energy particle acceleration during reconnection events take place over a macroscopic volume, rather than being confined to a narrow zone whose properties depend on microscopic transport coefficients.
\end{abstract}

\section{Introduction}

Turbulence is a common feature of astrophysical fluids. For instance, the interstellar medium (ISM) is known to be turbulent on scales ranging from AUs to kpc (see Armstrong et al., 1995; Elmegreen and Scalo, 2004; Lazarian, 2009 and ref. therein; Chepurnov and Lazarian, 2010). This is not surprising. Magnetized astrophysical plasmas generally have very large Reynolds numbers due to their large length scales. Laminar plasma flows at such high Reynolds numbers are prey to numerous linear and finite-amplitude instabilities, from which turbulent motions readily develop. Recent work on this topic is not about the existence of turbulence, but its properties, its effects and how to study turbulence in astrophysical settings (see Gaensler et al., 2011; Burkhart et al., 2012 for recent galactic studies; see Leamon et al., 1998; Bale et al., 2005 for the solar wind; Padoan et al., 2006 for molecular clouds; and Vogt and Enßlin, 2005; Enßlin and Vogt, 2006 for the intracluster medium).

The common textbook treatment of magnetic reconnection, i.e. the ability of magnetic field lines to change magnetic topology, treats the surrounding flow as negligible and the magnetic field lines as well-ordered, i.e. as though the surrounding turbulence did not exist. This is extremely problematic. Turbulence radically changes many astrophysical processes, e.g. diffusion, cosmic ray transport, advection of heat. It would be surprising if magnetic reconnection were not affected. 

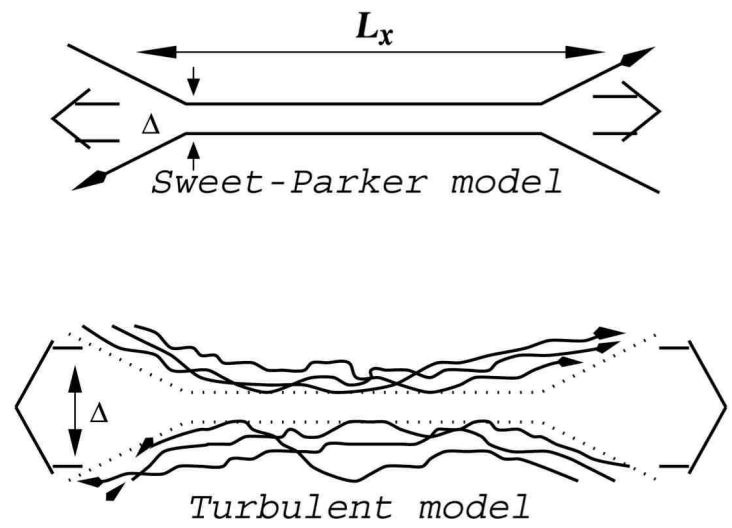

Fig. 1. Upper plot: Sweet-Parker model of reconnection. The outflow is limited by a thin slot, which is determined by Ohmic diffusivity. The other scale is an astrophysical scale $L_{x} \gg \Delta$. Lower plot: Reconnection of weakly stochastic magnetic field according to LV99. The Goldreich-Sridhar (1995) model of MHD turbulence is used to account for the stochasticity of magnetic field lines. The outflow in the LV99 theory is limited by the diffusion of magnetic field lines, which depends on field line stochasticity. From Lazarian et al. (2004).

Lazarian and Vishniac (1999) identified turbulent three dimensional field line wandering as the critical effect on magnetic reconnection speeds and quantified the expected reconnection rates (see Fig. 1). The LV99 model of turbulent reconnection is radically different from its predecessors. For instance, unlike Speiser (1970) and Jacobson and Moses (1984), the model does not appeal to changes in the microscopic properties of the plasma. The nearest progenitor to LV99 was the work of Matthaeus and Lamkin (1985) Matthaeus and Lamkin (1986), who studied the problem numerically in 2-D MHD and suggested that magnetic reconnection may be fast due to multiple $\mathrm{X}$ points and turbulent EMF. However, in contrast to LV99, these papers did not address the important role of magnetic field line wandering, and did not obtain a quantitative prediction for the reconnection rate. The predictions of LV99 have been successfully tested in Kowal et al. (2009).

In this paper we turn to the detailed structure of current sheets in a turbulent medium. To what extent is it reasonable to apply concepts taken from the study of homogeneous turbulence to the vicinity of a large scale magnetic field reversal? Are the local electric field strengths close to expectations for other models of reconnection? We will analyze typical time frames from the simulations of Kowal et al. (2009) to answer these questions.

\section{The current sheet in turbulent reconnection}

In Sweet-Parker reconnection (Parker, 1957; Sweet, 1958), the current sheet sits between two regions with strongly different magnetic field vectors, and the velocity field is smooth and highly symmetric. In particular the electric field perpendicular to the reconnection field component is just $\eta \boldsymbol{J}$. The large reconnection speed requires an anomalously large resistivity $\eta$. On the other hand, following LV99, we expect that in the presence of turbulence the velocity field will be rough, the current sheet will be highly distorted, and the electric field will be dominated everywhere by the advective term, $-\boldsymbol{v} \times \boldsymbol{B}$. What is less clear is the extent to which the current sheet maintains its identity amidst the turbulent flow.

The nature of the current sheet is interesting because it affects our understanding of the reconnection process, and our understanding of particle acceleration during reconnection events. In LV99 we assumed that the current sheet was welldefined and narrow. The surrounding turbulence was taken to have the properties of homogenous turbulence, despite the obvious dynamical importance of a narrow but large scale zone of very large magnetic shear. This picture was motivated by considering the turbulent fluid as a collection of magnetic field lines which evolve as well-defined dynamical entities with some intermittent distribution of reconnection events. In such a picture, the outflow zone from the large scale reconnection sheet may be large, but it is far from obvious that the shearing zone itself will diffuse.

In a reanalysis of this problem by Eyink et al. (2011), we presented a more rigorous derivation of the diffusion process. By treating the microscopic resistivity as a stochastic process it can be combined with a statistical treatment of the turbulence in a seamless manner. In this formalism the classical evolution of the magnetic field is recovered by taking the expectation value of the stochastic evolution. Individual field lines are no longer simple dynamical entities, in the sense that they can be defined exactly at any single time, but the identification of field lines from one time to another is no longer unique. The identity of a field line diffuses over time. This results in the same prediction for the diffusion of the outflow from a reconnection event as in the earlier picture, which is not surprising because formally the two methods are equivalent. However, in a qualitative sense, the latter picture suggests a somewhat different current sheet structure. If the identity of the field lines is not well-defined, and all properties diffuse, then the magnetic shearing zone will diffuse until the shear is spread over the width of the whole outflow.

In either case the outflow zone is not altogether like any similar volume in the turbulent medium. There will be a strong outflow, with some complicated local structure. This longitudinal flow will not couple strongly to the Alfvenic turbulence, but its precise effects are unclear.

In Kowal et al. (2009), we tested the predictions of LV99 by performing simulations in a box with open boundaries in the $\hat{x}$ direction, the direction of the reversing field component, periodic boundary conditions in the $\hat{z}$ (guide field) direction, and inflow boundary conditions in the $\hat{y}$ direction, perpendicular to the unperturbed current sheet. The simulations were run for a variety of parameters without turbulent 
(a)
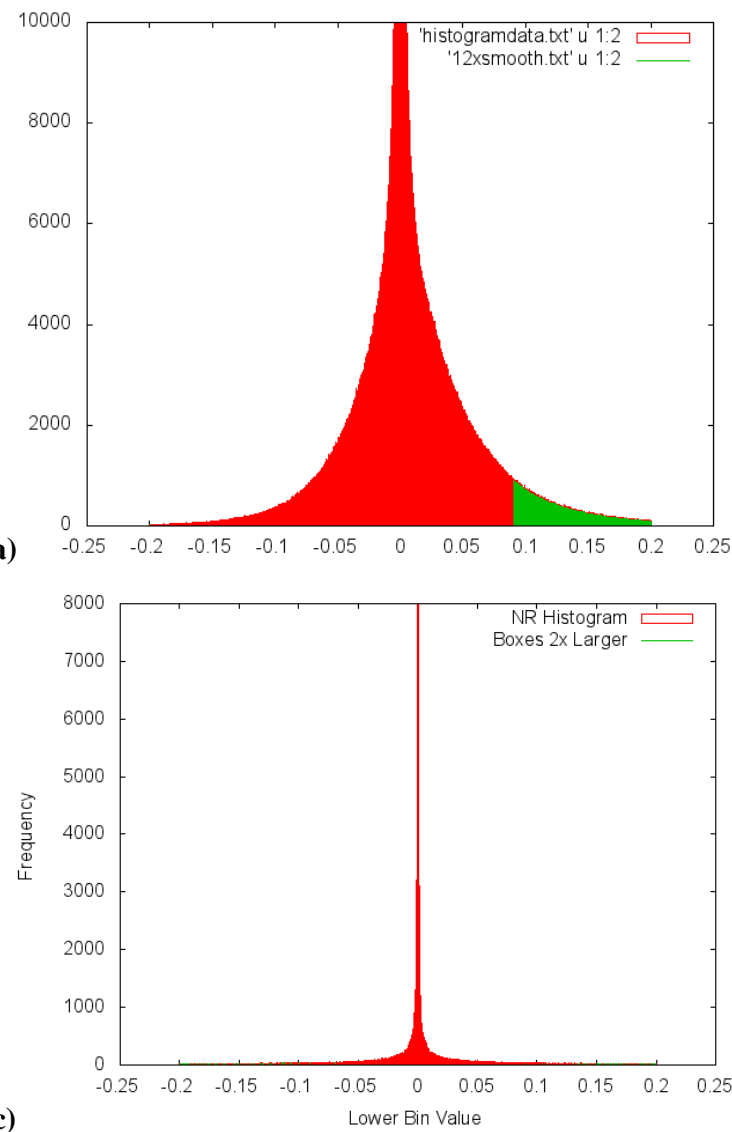
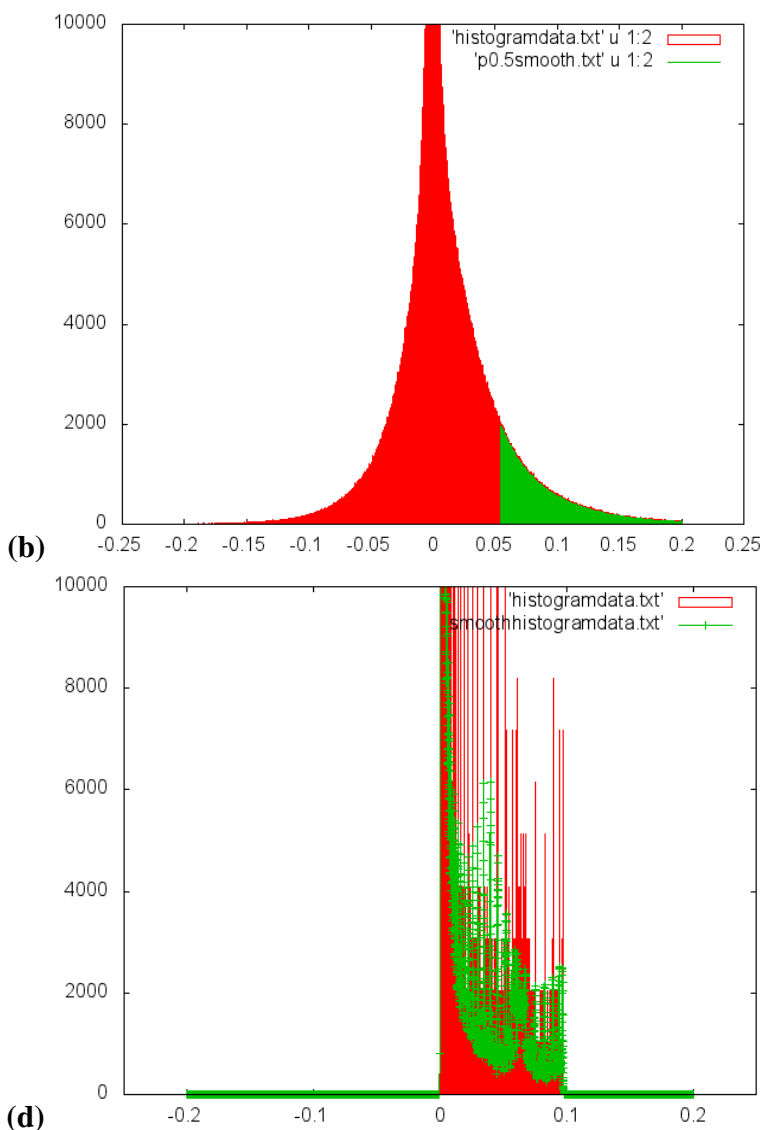

Fig. 2. These figures show histograms of the gradient of the reversing component of the large scale magnetic field in the direction normal to the unperturbed current sheet, i.e. $\partial_{y} B_{x}$. (a) is for the highest power simulation, $P=1$. (b) is for $P=0.5$. (c) is for $P=1$ but with no large scale magnetic field reversal, i.e. simply locally driven strong turbulence. Bins with twice the number of cells as the corresponding bin with the opposite sign of $\partial_{y} B_{x}$ are shown in green. (d) shows the first simulation in the absence of turbulent forcing.

forcing for several Alfvén times, in order to produce a SweetParker reconnection layer. Subsequently, sub-alfvenic turbulence was induced in the middle of the simulation volume via random forcing. This approach allowed us to verify that the boundary conditions were not biasing the reconnection process and to follow the evolution of the current sheet for an arbitrarily large number of Alfvén times. The simulations were run varying strengths of the guide field. (No differences were observed between simulations with a guide field equal to the reversing field component and a guide field smaller by a factor of 10.) The predictions of LV99 for the reconnection speed and its dependence on the amplitude of the turbulence and the size of the largest eddies were successfully tested against the average speed observed in the simulations. In particular, Kowal et al. (2009) confirmed that the turbulent reconnection speed was independent of the resistivity and the numerical resolution.

The structure of the current sheet was not addressed in Kowal et al. (2009). Here we will present some preliminary work based on the same numerical simulations discussed in that paper. In what follows, distances are measured in units of the box size, velocities in fractions of the Alfvén speed far from the reconnection zone, and time in units of $L_{\text {box }} / V_{A}( \pm \infty)$. In the first set of panels, we show a histogram of the $\partial_{y} B_{x}$ at $\mathrm{t}=12$ for three simulations, i.e. every cell in the simulation is assigned to a bin according to its $\partial_{y} B_{x}$. The fourth panel shows an early time in the simulation shown in Fig. 2a before the turbulent forcing has been turned on. The figures all use the same bin width, 0.00005 , but the last figure also shows a smoothed histogram in green, with each cell averaged with nine cells on either side. The vertical axis gives the number of cells in each bin. The first panel shows a run with an input power of 1 , in dimensionless units. The typical turbulent velocity as a fraction of the Alfvén speed is roughly $\sqrt{P / k_{\text {inj }}}$, where $k_{\text {inj }}$ is the wavenumber of the injected random forcing (normalized to unity for the fundamental mode of the box). The second panel has $P=0.5$ and the third panel has $P=1$, but with no large scale field reversal.

The first three figures are broadly similar, consisting in each case of a central peak around zero with wings extending smoothly, and falling monotonically, on both sides. The first 


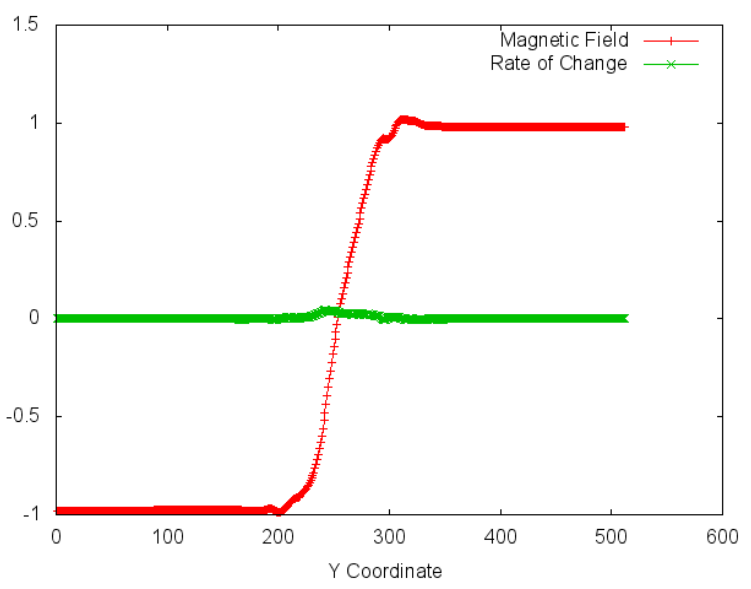

Fig. 3. The average magnetic field strength in the $\hat{x}$ (reversing) direction as a function of vertical distance in the computational box. The second line shows the field gradient.

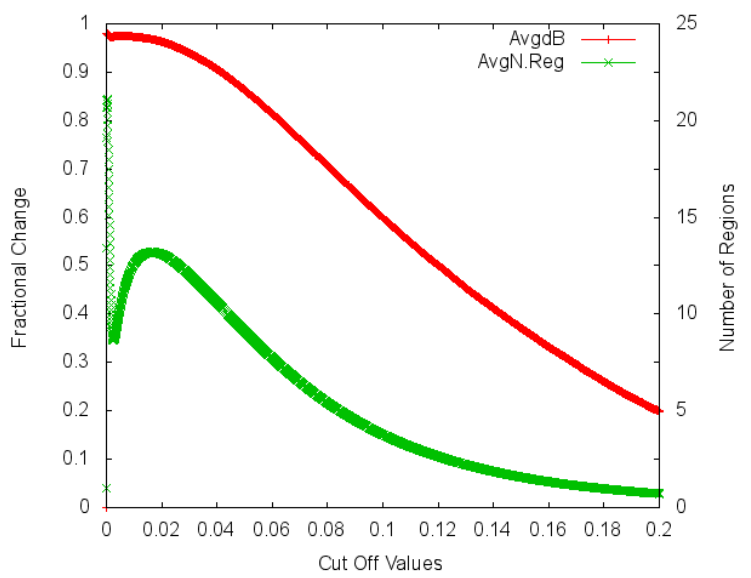

Fig. 4. The average number of regions with magnetic field gradients above a cutoff value, as a function of the cutoff value, and the fraction of the total change in the large scale magnetic field that falls within those regions.

two figures show a bias towards positive gradients, which is stronger for the weaker power cascade. In no case do we see a distinct feature showing a preferred value of the magnetic shear. We would expect such a feature if there were a large scale coherent structure in the current distribution, i.e. if the thin current sheet in the laminar case had been replaced by a similar, albeit distorted, feature embedded in the turbulence. Instead, the magnetic shear seems to be distributed among the individual turbulent eddies. We can contrast this with the fourth figure, which shows a nonturbulent current sheet. Ironically, this histogram is the most irregular, since the repetition of values from nearly identical points on the current sheet produces an extremely spiky distribution of points. Smoothing this by averaging each bin with the adjacent nine bins in both directions produces the green histogram. There we see the expected sharp cutoffs at high $\partial_{y} B_{x}$ and at zero, and a strong peak close to the upper cutoff that comes from the interior of the current sheet. As expected, none of these features are present in the other histograms.

Of course, this is just one way of looking at the structure of the current sheet. By focussing on the individual bins, we are ignoring the spatial relationship among them, which is a critical part of what we mean by a current sheet. In Fig. 3 we average the magnetic field strength over the $(x, z)$ plane and plot the result as a function of the $\hat{y}$-axis (normal to the unperturbed current sheet for the $P=1$ case). We see a broadened and smooth transition zone. There are relatively minor wiggles due to the turbulent eddies, which are largely erased by the averaging process.

We can gain more insight by considering the typical structures encountered passing through the transition zone. We can define a contiguous region by setting a cutoff for the absolute value of the magnetic field gradient. Adjacent cells for given $(x, z)$ that lie above the cutoff are grouped together. In Fig. 4 we show the average number of regions (marked on the left vertical axis) as a function of the cutoff value for the $P=1$ case. The red line shows the fraction of the total change in $B_{x}$ that occurs within the high gradient regions. The number of regions rises smoothly, peaking above 10, at which point virtually all the systematic change in $B_{x}$ is in one of the regions. For lower cutoff values the entire turbulent region becomes one contiguous structure. (The zeros in $B_{x}$ occur between grid points.) As the cutoff approaches zero we start to pick up very weak fluctuations outside the region of driven turbulence and the number of regions rises sharply before plunging to one.

We see from this figure that most of the systematic change in the magnetic field happens in a few contiguous regions, but not the single strongest one. Nor is there any obvious break in the distribution.

The remaining question is whether or not the structures identified by the cutoff level are discrete objects, or whether they are associated with each other and with extensive adjacent regions of slightly lower values of the magnetic field gradient. In Fig. 5a we plot the width of the reconnection layer marked by these structures, defined as the total distance from the leading edge of the first region to the trailing edge of the last. In Fig. 5b we plot the average width of the regions above the cutoff value. In both figures we also show the average number of regions. We see from these figures that the total width is quite large, comparable to the width of the outflow region when the bulk of the magnetic field change occurs within the individual regions. On the other hand, the average width of the contiguous regions of the cutoff is flat over a broad range of cutoff values. These regions are quite narrow, and not particularly associated with each other. 


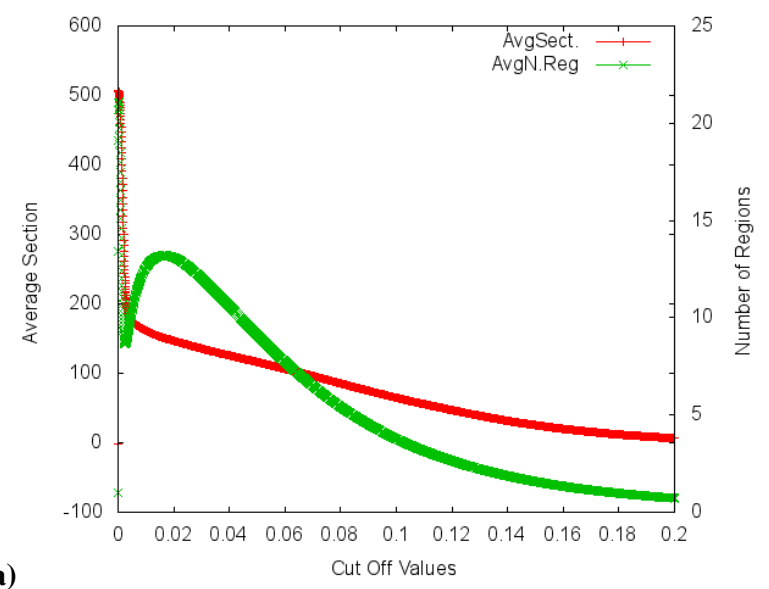

(a)

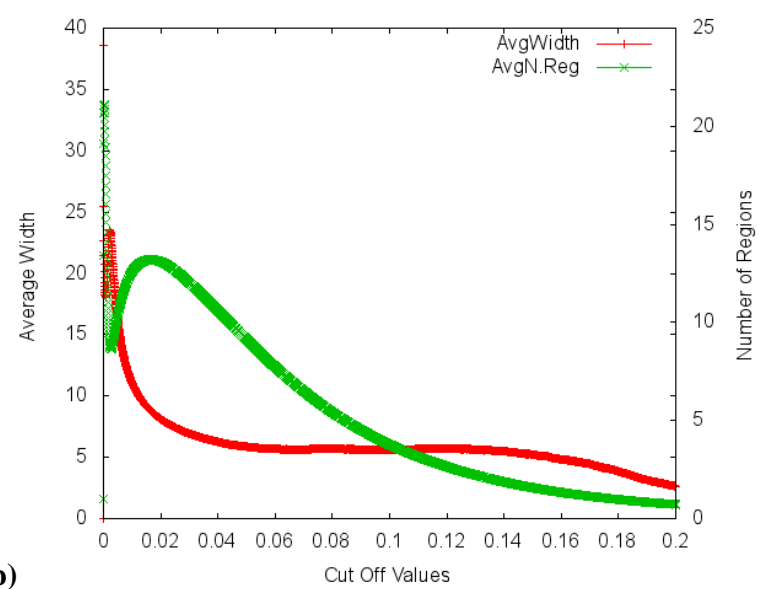

Fig. 5. (a) shows the width of the layer containing all the bins above the cutoff value of the magnetic field gradient averaged over the whole simulation box "average section". (b) shows the average width of each separate contiguous region above the cutoff.

\section{Conclusions}

We have analyzed the structure of the current sheets in simulations of large scale turbulent reconnection. Regions of strong current are narrow, but are spread throughout a broad layer, whose width is comparable to the outflow width. The change in the large scale magnetic field is small across any single current sheet and the distribution of current intensity is broadly similar to the distribution seen for comparable levels of turbulence but without any large scale magnetic field reversal. The distribution of current strengths is biased in the direction of the large scale current, but this manifests as a skewness in the distribution rather than a discrete feature.

Overall our results are consistent with the notion that turbulent reconnection is accomplished via many small scale current sheets. The presence of large scale magnetic shear biases the local turbulence, but does not seem to introduce any novel magnetic features.
Acknowledgements. The work of ETV and SP is supported by the National Science and Engineering Research Council of Canada. The research of GK is supported by FAPESP grant no. 2009/50053-8, AL is supported by the Center for Magnetic Self-Organization in Laboratory and Astrophysical Plasmas, NSF grant AST-08-08118 and NASA grant NNX09AH78G. GE was partially supported by NSF grants AST 0428325 and CDI-II: CMMI 0941530. This research also was supported by the National Science Foundation project TG-AST080005N through TeraGrid resources provided by Texas Advanced Computing Center (TACC:http://www.tacc.utexas.edu). Part of this work was made possible by the facilities of the Shared Hierarchical Academic Research Computing Network (SHARCNET:http://www.sharcnet.ca) and the GALERA supercomputer in the Academic Computer Centre in Grańsk (TASK:http://www.task.gda.pl).

Edited by: T. Intrator

Reviewed by: two anonymous referees

\section{References}

Armstrong, J. W., Rickett, B. J., and Spangler, S. R.: Electron density power spectrum in the local interstellar medium, Astrophys. J., 443, 209-221, 1995.

Bale, S. D., Kellogg, P. J., Mozer, F. S., Horbury, T. S., and Reme, H.: Measurement of the Electric Fluctuation Spectrum of Magnetohydrodynamic Turbulence, Phys. Rev. Lett., 94, 215002, doi:10.1103/PhysRevLett.94.215002, 2005.

Burkhart, B., Lazarian, A., and Gaensler, B. M.: Properties of Interstellar Turbulence from Gradients of Linear Radio Polarization Maps, Astrophys. J., 749, 145 (16 pp.), 2012.

Chepurnov, A. and Lazarian, A.: Extending the Big Power Law in the Sky with Turbulence Spectra from Wisconsin $\mathrm{H} \alpha$ Mapper Data, Astrophys. J., 710, 853-858, 2010.

Elmegreen, B. G. and Scalo, J.: Interstellar Turbulence I: Observations and Processes, Annu. Rev. Astron. Astrophys., 42, 211273, 2004.

Enßlin, T. A. and Vogt, C.: Magnetic turbulence in cool cores of galaxy clusters, Astron. Astrophys., 453, 447-458, 2006.

Eyink, G. L., Lazarian, A., and Vishniac, E. T.: Fast Magnetic Reconnection and Spontaneous Stochasticity, Astrophys. J., 743, 51 (28 pp.), 2011.

Gaensler, B. M., Haverkorn, M., Burkhart, B., Newton-McGee, K. J., Ekers, R. D., Lazarian, A., McClure-Griffiths, N. M., Robishaw, T., Dickey, J. M., and Green, A. J.: Low-Mach-number turbulence in interstellar gas revealed by radio polarization gradients, Nature, 478, 214-217, 2011.

Goldreich, P. and Sridhar, S.: Toward a theory of interstellar turbulence, 2: Strong alfvenic turbulence, Astrophys. J., 438, 763-775, 1995.

Jacobson, A. R. and Moses, R. W.: Nonlocal dc electrical conductivity of a Lorentz plasma in a stochastic magnetic field, Phys. Rev. A, 29, 3335-3342, 1984.

Kowal, G., Lazarian, A., Vishniac, E. T., and Otmianowska-Mazur, K.: Numerical Tests of Fast Reconnection in Weakly Stochastic Magnetic Fields, Astrophys. J., 700, 63-85, 2009.

Lazarian, A.: Obtaining Spectra of Turbulent Velocity from Observations, Space Sc. Rev., 143, 357-385, 2009. 
Lazarian, A. and Vishniac, E. T.: Reconnection in a Weakly Stochastic Field, Astrophys. J., 517, 700-718, 1999. (LV99)

Lazarian, A., Vishniac, E. T., and Cho, J.: Magnetic Field Structure and Stochastic Reconnection in a Partially Ionized Gas, Astrophys. J., 603, 180-197, 2004.

Leamon, R. J., Smith, C. W., Ness, N. F., Matthaeus, W. H., and Wong, H. K.: Observational constraints on the dynamics of the interplanetary magnetic field dissipation range, J. Geophys. Res., 103, 4775-4787, doi:10.1029/97JA03394, 1998.

Matthaeus, W. H. and Lamkin, S. L.: Rapid magnetic reconnection caused by finite amplitude fluctuations, Phys. Fluids, 28, 303307,1985 .

Matthaeus, W. H. and Lamkin, S. L.: Turbulent magnetic reconnection, Phys. Fluids, 29, 2513-2534, 1986.
Padoan, P., Juvela, M., Kritsuk, A., and Norman, M. L.: The Power Spectrum of Supersonic Turbulence in Perseus, Astrophys. J. Lett., 653, L125-L128, 2006.

Parker, E. N.: Sweet's Mechanism for Merging Magnetic Fields in Conducting Fluids, J. Geophys. Res., 62, 509-520, 1957.

Speiser, T. W.: Conductivity without collisions or noise, Plan. Space Sci., 18, 613-1970.

Sweet, P. A.: The Neutral Point Theory of Solar Flares, in: Electromagnetic Phenomena Conf. Proc. IAU Symposium 6, edited by: Lehnert, B.,Electromagnetic Phenomena in Cosmical Physics, (Cambridge, UK:Cambridge University Press), Cosmical Physics, 6, 123-134, 1958.

Vogt, C. and Enßlin, T. A.: A Bayesian view on Faraday rotation maps Seeing the magnetic power spectra in galaxy clusters, Astron. Astrophys., 434, 67-76, 2005. 